\title{
Particle Velocity Measurement of Pulverized Coal flow on a Power Plant Using Electrostatic Sensor Array
}

\author{
Jingyu Zhang ${ }^{+}$, Liguo Tian and Meng Li \\ Tianjin Key Laboratory of Information Sensing and Intelligent Control, Tianjin University of Technology \\ and Education, Tianjin 300222, China
}

\begin{abstract}
The particle velocity of pulverized coal in pneumatic conveying pipelines is a key parameter directly related to a higher efficiency and reducing energy consumption of coal-fired power plant. The experiment was conducted on a coal-fired power plant. The cross-correlation velocities between different electrode pairs from electrostatic sensor arrays were presented in this paper. It was found that there was little difference in the particle velocity between the top and bottom areas, which indicated that the stratification flow in the pipeline was not obvious. However the distributions of particle velocities at left and right areas were not uniform. This was due to the influence of the downstream elbow. It can be confirmed that the particle velocities of pulverized coal at different positions in the pipeline are uneven, and a more detailed measurement method need be applied.
\end{abstract}

Keywords: electrostatic sensor array, particle velocity measurement, pulverized coal, coal-fired power plant.

\section{Introduction}

Multi-phase flows have been widely applied in coal-fired power industries. For example, the pulverized coal in pneumatic conveying pipelines is dilute gas-solid two-phase flow, and the particle velocity is a key parameter directly related to a higher efficiency and reducing energy consumption. However, the flow field distribution in the pulverized coal flow is complex, and the particle velocities at different locations may be inhomogeneous [1]. Thus, the accurate measurement of particle velocities at different locations is important in studying the measurement mechanism of pulverized coal flow [2].

The particle velocity measurement method using electrostatic sensor array (ESA) can be classified into three categories, namely local velocity measurement method in near-electrode region, tomography method and cross-correlation focus method. The former is based on the local sensitivity of electrostatic electrode, so only the velocity in the area near electrode may be detected. Xu et al applied the spatial filtering method to measure local velocities of pneumatically conveyed particles [3]-[5]. Qian et al used ipsilateral electrode pairs to measure the local velocity of biomass and blended biomass fuels in pneumatic conveying pipelines [6] and pulverized coal flow in a power plant [7]. Zhang et al applied the cross-correlation method between ipsilateral electrode pairs in a triple-bed combined circulating fluidized bed [8].The tomography method needs an electrostatic tomography system to reconstruct the particle concentration distribution, and then uses the pixel-to-pixel correlation method to calculate the velocity distribution [9]-[14]. Green et al introduced linear projection method for the image reconstruction [10-11], while Rahmat et al introduced filter backprojection method [13] and least square with regularization method [14]. However, the reconstruction methods typically faced ill-posed problem and their solutions were unstable and inaccurate [14]. The crosscorrelation focus method makes full use of ipsilateral, adjacent and contralateral electrode pairs, which could get information about particle velocity in more regions than that only in near-electrode region [15].

\footnotetext{
+ Corresponding author. Tel.: + 86 15822943148; fax: +86 02288181529.

E-mail address: zhangjingyu@tju.edu.cn.
} 
The experiment was conducted on a coal-fired power plant. The inner diameter of the experimental pneumatic conveying pipelines is $460 \mathrm{~mm}$ and the air velocity is from 20 to $30 \mathrm{~m} / \mathrm{s}$, which will leads to inhomogeneous particle distribution. The cross-correlation velocities between different electrode pairs were presented in this paper to verify the particle velocity distribution.

\section{Experimental Scheme}

\subsection{Coal-fired power plant experiment}

When a coal-fired power plant works, the fuel coal must first be prepared into pulverized coal particles in the mills, and then be fed into the boiler by pneumatic conveying pipeline for combustion. Thus the particle size, concentration and conveying velocity of pulverized coal will have an impact on the operation of the boiler system. When the pulverized coal with wide particle size distribution is transported in horizontal and vertical pipes, the distribution of its concentration and velocity in the cross section of the same pipeline will be inhomogeneous, possibly lead to the pipe blockage accident. On the other hand, the particle velocity of pulverized coal also has a great influence on combustion. If the pulverized coal velocity is too low in the boiler, the flame will be too close to the nozzle and burn the boiler. If the pulverized coal velocity is too high, some pulverized coal particles will leave the combustion boiler without complete combustion, increasing the mechanical loss of the boiler and facing the risk of coking on the water-cooling wall.

Figure 1 shows the schematic diagram of coal-fired power plant. The electrostatic sensor arrays were installed on the four pneumatic conveying pipelines from each mills, in which the pulverized coal is tangentially blown into the boiler. Figure 2 shows the installation location of electrostatic sensor arrays on 1\# pipes from mill A to mill $\mathrm{E}$. The electrostatic signals were transferred by the conditioning circuits and then sampled by the A/D modules. The cross-correlation velocities were calculated by the DCS computer.

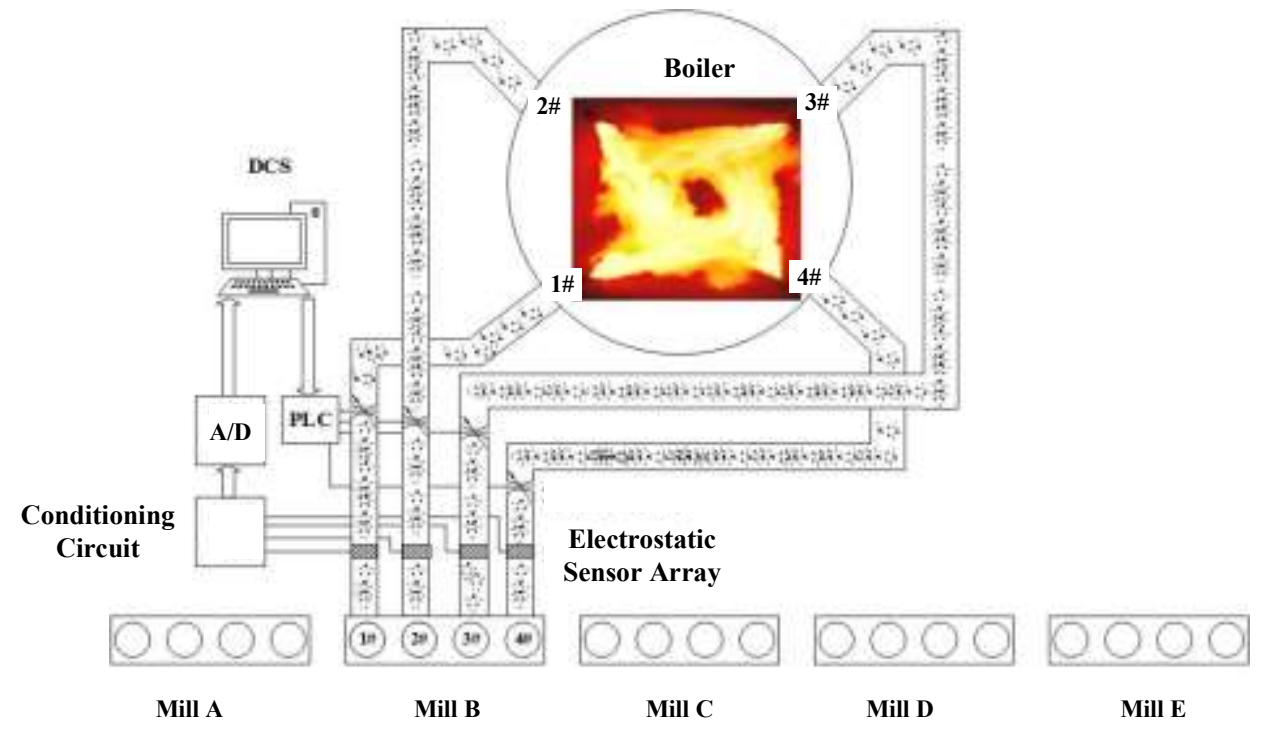

Fig. 1: Schematic Diagram of Coal-Fired Power Plant.

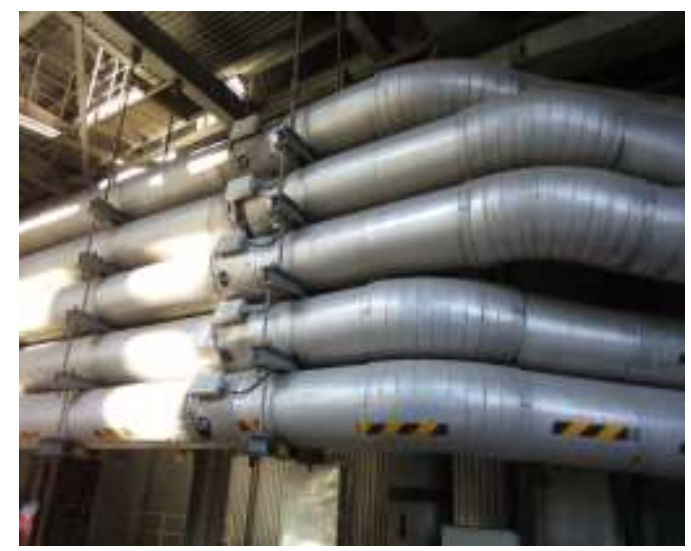

Fig. 2: Installation Location of Electrostatic Sensor Arrays. 


\subsection{Electrostatic sensor array}

Figure 3 shows the structure of electrostatic sensor array. Electrostatic sensor array is composed of the arc-shaped and ring-shaped electrode array, the insulating layer, the pipe wall, the circuit bases and the flanges. There are 8 arc-shaped electrodes installed on two sections (section A and B). The electrodes are respectively near the top, the right, the bottom and the left area of the pipeline. The distance between section upstream and downstream is $50 \mathrm{~mm}$. The width of an electrode is $6 \mathrm{~mm}$, and the radian is 60 degree. There are 2 ring-shaped electrodes installed on two sections, too. The distance between them is $50 \mathrm{~mm}$. The width of an electrode is $6 \mathrm{~mm}$. The insulating layer is between the electrodes and the pipe walls.

When charged particles move through the electrode, certain charge is induced on the inside wall of the electrode. The outside wall of the electrode is connected to the conditioning circuit, so that the induced current is converted to a voltage signal.

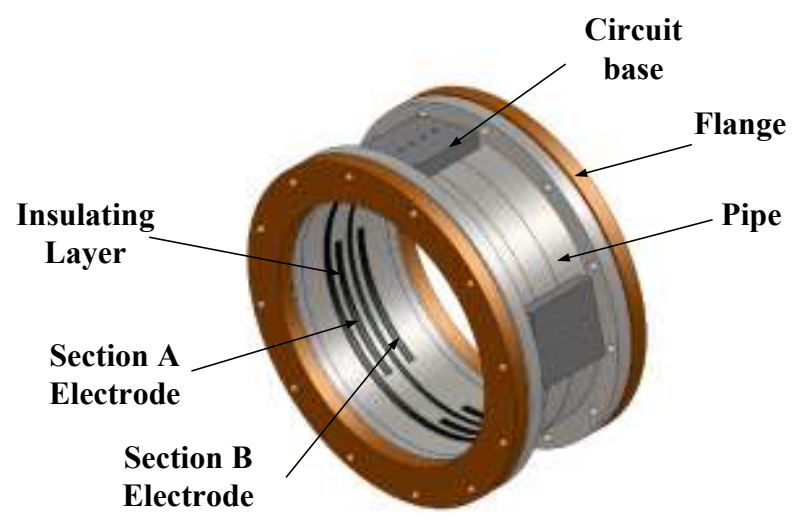

Fig. 3: Electrostatic Sensor Array.

\subsection{Cross-correlation method}

The spatial sensitivity of an electrostatic electrode is defined as the absolute value of the induced charge on the electrode when a unit point charge is positioned in the sensing zone of the sensor [15]. The spatial sensitivity $s$ can be determined as

$$
s(x, y)=\left|\frac{q}{e(x, y)}\right|
$$

where $q$ is the induced charge on the electrode, $e$ is the point charge and $(x, y)$ is the position of it.

The cross-correlation velocity between signals derived from different electrode pairs on two sections can be obtained as [16]

$$
v_{i j}=\frac{L}{\tau_{i j}}=\frac{\iint \sigma_{A}^{2}(x, y) s_{A_{i}}(x, y) s_{B_{j}}(x, y) d x d y}{\iint \frac{1}{v_{p}(x, y)} \sigma_{A}^{2}(x, y) s_{A_{i}}(x, y) s_{B_{j}}(x, y) d x d y}
$$

where $s_{A_{i}}$ and $s_{B_{j}}$ are the spatial sensitivity distribution of the electrode $A_{i}$ and $B_{j}$ respectively. $\sigma_{A}$ and $\sigma_{B}$ are the charge density distribution on section $A$ and $B$ respectively. $v_{p}$ is the distribution of particle velocity.

Based on the local sensitivity of arc-shaped electrostatic electrode, the cross-correlation sensitivity between signals derived from different electrode pairs have different focus areas. The Cross-correlation velocity between ipsilateral electrode pair primarily represents the velocities of the particles in the nearelectrode region. 


\section{Experimental Results and Analysis}

During the trial operation of the power plant, the air velocity is measured by the air flowmeter, and the Klinzing model [16] is used to estimate the pulverized coal velocity. The velocity of pulverized coal in different pipelines was measured by electrostatic sensor arrays, and the results were compared with those estimated by Klinzing model.

Table 1: Particle Velocity Measurement Results of Four Pipes from mill B

\begin{tabular}{ccccc}
\hline Pipe & $1 \#$ & $2 \#$ & $3 \#$ & $4 \#$ \\
\hline Air velocity $(\mathrm{m} / \mathrm{s})$ & 28.66 & 26.36 & 25.01 & 22.26 \\
Estimated particle velocity $(\mathrm{m} / \mathrm{s})$ & 26.95 & 24.78 & 23.50 & 20.90 \\
Measured particle velocity $(\mathrm{m} / \mathrm{s})$ & 25.95 & 24.01 & 22.58 & 19.88 \\
Relative error $(\%)$ & 3.85 & 3.21 & 4.07 & 5.13 \\
\hline
\end{tabular}

Particle velocity measurement results of four pipes from mill B is shown in Table 1. It was found that the particle velocities of four pipes from the same mill were different. The particle velocity in 1\# pipe was the highest and the particle velocity in 4\# pipe was the lowest. The uneven particle velocity of the four pipes would lead to inclined flame, which was dangerous. At the same time, the estimated value of particle velocity using Klinzing model had larger errors when the air velocity was lower. Because the flow would be inhomogeneous in a section when the air velocity became slow.

The cross-correlation velocity between ipsilateral electrode pair is shown in figure 4 . It can be found that the average particle velocity at the top area was $25.9 \mathrm{~m} / \mathrm{s}$, and that at the bottom area was $25.0 \mathrm{~m} / \mathrm{s}$. There was little difference in the particle velocity between the top and bottom regions, which indicated that the stratification flow in the pipeline was not obvious. The particle velocity at the left area was $26.4 \mathrm{~m} / \mathrm{s}$, and that of the right area was $24.1 \mathrm{~m} / \mathrm{s}$. The distribution of particles in the pipeline at left and right areas was not uniform. This was due to the influence of the downstream elbow. The left electrode was close to the inside of the elbow, while the right electrode was close to the inside of the elbow. Thus the particle velocity inside the elbow is higher than that outside the elbow.

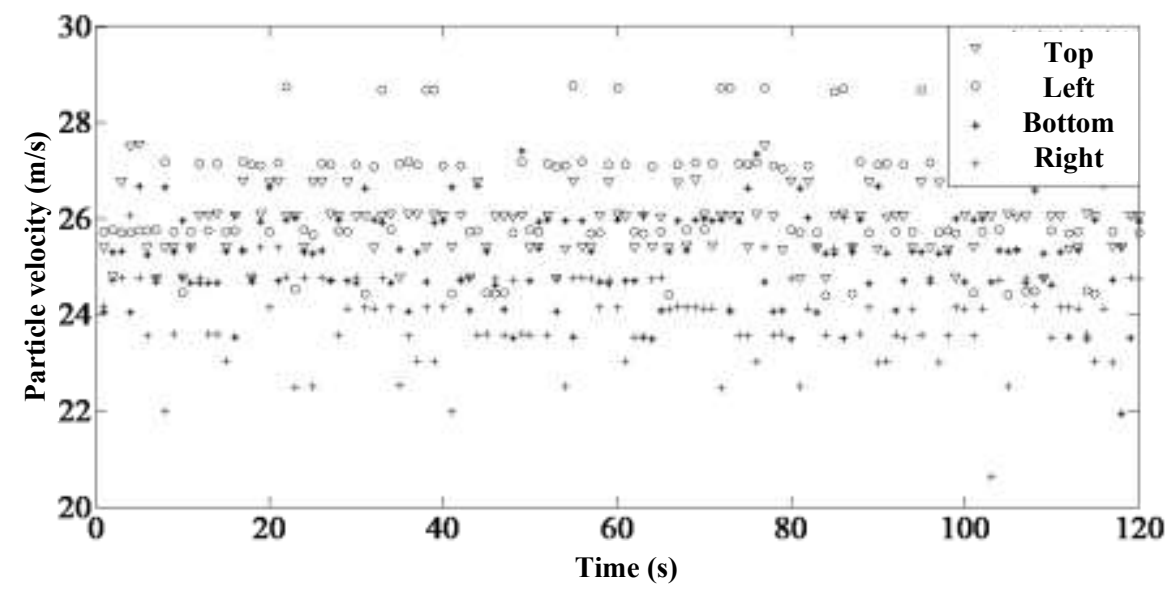

Fig. 4: Particle velocities at different location in a pipes.

\section{Conclusions}

This experimental measurement was on the pulverized coal conveying pipeline on a coal-fired power plant. Through the measurement results, it can be confirmed that the particle velocities of pulverized coal at different positions in the pipeline are uneven, and it is easily affected by the elbow. A more detailed measurement method need be applied if it is used for industrial closed loop control.

\section{Acknowledgements}

This work is supported by the Natural Science Foundation of Tianjin under Grants 18JCQNJC74500 and Scientific Research Fund in Tianjin University of Technology and Education under Grants KJ1732 and KRKC011815 and the Program for Discipline Leading Talents. 


\section{References}

[1] Chao W, Zhang J, Zhang Y, et al. Representation of induced and transferred charge in the measurement signal from electrostatic sensors. Instru. \& Meas. Technol. Conference. 2014: 1534-1538.

[2] $\mathrm{Xu} \mathrm{C}$, Li J, Gao H and Wang S. Investigations into sensing characteristics of electrostatic sensor arrays through computational modelling and practical experimentation. J. Electrostat. 2012, 70: 60-71.

[3] Li J, Xu C and Wang S. Local particle mean velocity measurement using electrostatic sensor matrix in gas-solid two-phase pipe flow. Flow Meas. Instrum. 2012, 27: 104-112.

[4] Li J, Xu C and Wang S. Spatial filtering characteristics of electrostatic sensor matrix for local velocity measurement of pneumatically conveyed particles. Measurement 2014, 53: 194-205.

[5] Qian X and Yan Y. Flow measurement of biomass and blended biomass fuels in pneumatic conveying pipelines using electrostatic sensor-arrays. IEEE T. Instrum. Meas. 2012, 61: 1343-1352.

[6] Qian X, Huang X, Hu Y and Yan Y. Pulverized coal flow metering on a full-scale power plant using electrostatic sensor arrays Flow Meas. Instrum. 2014, 40: 185-191.

[7] Zhang W, Cheng Y, Wang C, Yang W and Wang C H. Investigation on hydrodynamics of triple-bed combined circulating fluidized bed using electrostatic sensor and electrical capacitance tomography. Ind. Eng. Chem. Res. 2013, 52: 11198-11207.

[8] Thuku T I, Rahmat M F, Abdul W N, Tajdari T and Amuda Y A. 2-D finite-element modeling of electrostatic sensor for tomography system. Sensor Rev. 2013, 33: 104-113.

[9] Wang C, Zhang J, Zheng W, et al. Signal decoupling and analysis from inner flush-mounted electrostatic sensor for detecting pneumatic conveying particles. Powder Technol. 2017, 305: 197-205.

[10] Wang C, Zhan N, Jia L, et al. DWT-based adaptive decomposition method of electrostatic signal for dilute phase gas-solid two-phase flow measuring. Powder Technol. 2018, 329: 199-206.

[11] Thuku I T and Rahmat M F. Finite-element method modeling in 4 and 16 sensors electric-charge tomography systems for particles moving in pipeline. Flow Meas. Instrum. 2014, 38: 9-20

[12] Yan Y, Wang L, Wang T, et al. Application of soft computing techniques to multiphase flow measurement: A review. Flow Meas. Instrum. 2018, 60: 30-43.

[13] Yang Y, Zhang Q, Zi C, et al. Monitoring of particle motions in gas-solid fluidized beds by electrostatic sensors. Powder technol. 2017, 308: 461-471.

[14] Zheng Y and Liu Q. Review of techniques for the mass flow rate measurement of pneumatically conveyed solids. Measurement, 2011, 44: 589-604

[15] Wang C, Zhang J, Gao W, et al. Cross-correlation focus method with an electrostatic sensor array for local particle velocity measurement in dilute gas-solid two-phase flow. Meas. Sci. Technol. 2015, 26(11):115301-115310.

[16] Sun J, Yan Y. Non-intrusive characterisation of particle cluster behaviours in a riser through electrostatic and vibration sensing. Chem. Eng. Jour. 2017, 323: 381-395. 